\title{
Reaction of Analyte Ions with Neutral Chemical Ionization Gas
}

\author{
Kevin J. Hart, Scott A. McLuckey, and Gary L. Glish \\ Analytical Chemistry Division, Oak Ridge National Laboratory, Oak Ridge, Tennessee, USA
}

\begin{abstract}
Ion-molecule reactions of neutral methane with analyte ions under normal methane chemical ionization conditions are discussed. Reactant ions can be generated by direct electron ionization (EI) fragmentation, chemical ionization (CI) fragmentation, or collision-induced dissociation (CID). Examples in which products of such reactions appear in mass spectra in both conventional $\mathrm{CI}$ sources in "beam" instruments and low pressure $\mathrm{Cl}$ in a quadrupole ion trap are presented. Also shown is an example in which MS/MS product ions react with neutral methane used for $\mathrm{CI}$ in an ion trap. It is shown that it is relatively straightforward to recognize such reactions in a quadrupole ion trap and in certain cases to minimize or preclude them. Effects of various operating parameters have been investigated and are discussed. (J Am Soc Mass Spectrom 1992, 3, 549-557)
\end{abstract}

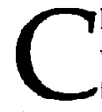
hemical ionization (CI) is one of the most widely used ionization techniques. Some of the attributes of CI that make it popular are that it generally imparts limited internal energy to the analyte upon ionization and thus minimizes fragmentation, the internal energy deposited upon ionization can be controlled via the reaction thermochemistry, it has good sensitivity, and it can be selective [1]. Chemical ionization is predicated on ion-molecule reactions. The most common CI reaction is proton transfer to give a protonated analyte molecule, but a number of reagent gases can give other types of analyte ions. The reagent gas is typically chosen to favor a particular reaction, such as proton transfer or electron transfer. However, other reactions may also proceed to a significant extent under $\mathrm{CI}$ conditions. In conventional $\mathrm{CI}$ on a beam instrument, for example, reactions involving the ion source surface or a variety of species in the CI plasma, such as electrons, radicals, and excited neutrals, can also occur [2]. Many of these reactions are competitive with the intended ionization reaction. It is perhaps less widely recognized that consecutive reactions can also significantly affect the CI mass spectrum. One such reaction, highlighted here, is the reaction of an analyte ion with the neutral reagent gas, subsequent to the formation of the analyte ion from an ionization event such as a reaction between the reagent ion and a neutral analyte. This type of reaction often goes unrecognized, particularly when the charged product of the reactions falls at an $m / z$ value that might be expected from the analyte

Address reprint requests to Gary L. Glish, Analytical Chemistry Division, Oak Ridge National Laboratory, Oak Ridge TN 37831-6365. molecule. Also, ordinarily, no simple experiment is available to alert the mass spectrometrist to the presence of such reactions.

A pressure of $0.1-1.0$ torr of reagent gas is usually maintained in the source of quadrupole or sector beam instruments to conduct $\mathrm{CI}$ experiments. With typical rate constants for $\mathrm{Cl}$ ion-molecule reactions of $10^{-9}-10^{-11} \mathrm{~cm}^{3} /$ molecule-s and ion source residence times (reaction times) of tens to hundreds of microseconds, easily measurable ion currents are generated for analytes at pressures in the range of $10^{-7}-10^{-8}$ torr or less. The high reagent gas pressure is necessary to provide sufficient number densities of reagent ions. At lower reagent ion densities, as in the ion trap, much longer reaction times are required to achieve comparable sensitivities [3]. Due to space charge limitations in ion-trapping instruments, the reagent ion density is several orders of magnitude lower than in CI sources on beam instruments, However, in trapping instruments the reaction time can be readily controlled, and thus reaction times from tens of milliseconds up to several seconds can be used to compensate for the low number density of reagent ions.

In this article we discuss reactions of analyte ions with the neutral reagent gas and demonstrate advantages of quadrupole ion traps, as compared to beam instruments with conventional $\mathrm{Cl}$ ion sources, for recognizing and in some situations minimizing or precluding such reactions. Three distinct pathways by which the reactant ion can be formed are discussed: ionization or fragmentation during the electron pulse used to generate the CI reagent ion or ions, fragmentation from the chemical ionization reaction, and collision-induced dissociation in MS/MS experiments. The 
first two of these occur in both ion traps and beam CI sources. Ion-trapping instruments, however, provide more options to minimize or at least identify these types of reactions. The reactions of ions with the $\mathrm{CI}$ reagent gas during MS/MS is much more likely to occur in ion-trapping instruments than in beam instruments. This results from ionization and analysis being done in the same region, separated in time, not in space as in beam instruments.

\section{Experimental}

\section{Reagents and Samples}

All CI spectra were acquired using high purity grade methane (Scott Specialty Gases, Baton Rouge, LA, 99.99\%; Aldrich Chemical Co., Milwaukee, WI, $>99.0 \%$ ) as the reagent gas at the indicated pressures listed in the text. Analytes included acetophenone, chlorobenzene, and p-dichlorobenzene (Chem Service, West Chester, PA, 99\%). Helium was used as the buffer and CID gas in the ion trap at a pressure of $7 \times 10^{-4}$ torr. All pressures reported for the ion trap mass spectrometer were measured with a GranvillePhillips model 280 ionization gauge and corrected using the method of Bartmess and Georgiadis [4]. Argon was used as the CID gas on the TSQ-700 at an indicated pressure of $7 \times 10^{-4}$ torr. This pressure and the source pressure on the TSQ-700 are actually measured on the gas inlet lines with Granville-Phillips Convectron, cat. no. 275071. The actual CI source pressure in the TSQ was estimated by using the $43^{+} / 57^{+}$ratio from isobutane [5].

\section{Instrumentation}

The quadrupole ion trap used for these experiments was the Finnigan MAT (San Jose, CA) ion trap mass spectrometer (ITMS). Samples were introduced into the ITMS via a precision leak valve (Varian Inc., model 951-5106). Chemical ionization mass spectra were acquired by ionizing at a radiofrequency (RF) amplitude corresponding to a low mass cutoff of 3.6 or $14.0 \mathrm{u}$, as noted in the text. A $10-\mathrm{ms}$ reaction time was used to allow the appropriate reagent ions to be formed and interact with the neutral analyte molecules, unless otherwise noted in the text. This reaction time is the sum of the part of the scan function denoted as "reaction time" (either $\mathrm{Cl}$ or MS/MS) at a fixed RF value plus the time during the analytical $\mathrm{RF}$ ramp prior to ejection of the reactant ion. Since ions must be ejected from the ITMS into the detector by order of increasing $\mathrm{m} / z$ at a rate of $180 \mu \mathrm{s} / \mathrm{u}$, an analytical scan starting at $20 \mathrm{u}$ will allow $10.26 \mathrm{~ms}$ for the $m / z 77$ ion to react with the neutral methane before it is ejected. However, if the analytical scan is started at $50 \mathrm{u}$ rather than $20 \mathrm{u}$, only 4.86 ms elapses prior to ejection of $m / z 77$. The change in reaction time during the analytical scan is illustrated

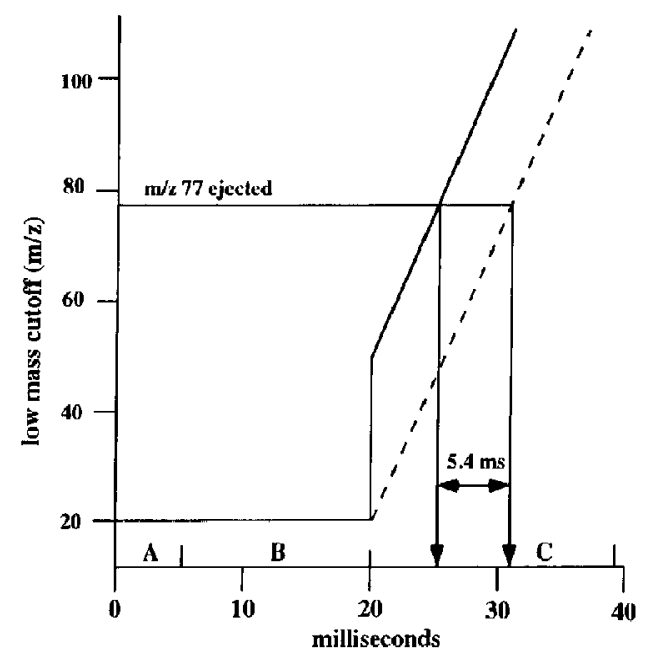

Figure 1. Two ITMS scan functions showing an analytical ramp from $20 \mathrm{u}$ (dashed line), $50 \mathrm{u}$ (solid line), and the difference in elapsed time for ejection of $m / z$ 77. The major regions of the scan functions are $(A)$ the ionization pulse, $(B)$ the reaction time, and $(C)$ the analytical ramp.

in a typical scan function in Figure 1. Selected reagent ion chemical ionization (SRICI) [6] was performed by first forming the reagent ions during a short reaction period and then isolating a specific reagent ion using the main $R F$ voltage and the dc voltage. A second, variable reaction period allows the selected reagent ions time to react with the neutral analyte molecules. MS/MS spectra were acquired in the conventional manner [7]. Briefly, this entails isolating the parent ion using the RF and dc voltages as before and then resonantly exciting the parent ion with an auxiliary RF "tickle" voltage applied to the endcaps of the ion trap.

Experiments using conventional $\mathrm{CI}$ in a beam instrument employed a Finnigan MAT TSQ-700. This is a tandem quadrupole mass spectrometer with an octapole serving as the collision region. Chemical ionization mass spectra were acquired on this instrument using the first quadrupole as the mass filter and operating the octapole and second quadrupole mass filter in RF-only mode (i.e., the Q1MS scan mode). The reagent gas pressure was varied using the needle valve provided with the instrument. The MS/MS spectra were obtained by admitting argon into the octapole to a pressure of $0.7 \mathrm{mtorr}$ and scanning the last quadrupole to obtain a CID product spectrum.

\section{Results and Discussion}

Three different pathways for generating ions that are reactive with neutral methane (used as a CI reagent gas) are discussed. In most cases, compounds that generate the phenyl cation, $\mathrm{C}_{6} \mathrm{H}_{5}^{+}$, are used as examples. This ion reacts with neutral methane according 
to eq 1:

$$
\mathrm{C}_{6} \mathrm{H}_{5}^{+}+\mathrm{CH}_{4} \rightarrow \mathrm{C}_{7} \mathrm{H}_{7}^{+}+\mathrm{H}_{2}
$$

(There have been several measurements of the rate constant of this reaction [8-11]. The product ion has been reported to have the tropylium structure [11]; however, other experiments we have performed suggest it to be benzyl. These results will be reported elsewhere [12].) Although this particular reaction is used throughout this article for illustrative purposes, many other ions are capable of reacting with neutral methane or other reagent gases. For example, some substituted phenyl cations are known to react with neutral methane [13], and we have observed these reactions in the ion trap. Unsaturated hydrocarbon ions are also known to react readily with hydrocarbons [14] commonly used for CI, and this too has been observed. It should be emphasized, though, that such reactions are not unique to $\mathrm{Cl}$ in an ion trap; they will also occur in conventional beam instrument $\mathrm{Cl}$ sources as shown below.

\section{Reaction with Analyte lons Formed during EI of Reagent Gas}

The first step in any chemical ionization experiment is the ionization of the reagent gas. This step is generally accomplished by electron ionization, and thus some of the analyte molecules will also be ionized by the electron beam. The phenyl cation, $\mathrm{C}_{6} \mathrm{H}_{5}^{+}$, is a common EI fragment for monosubstituted benzene compounds. The EI mass spectrum of acetophenone $\left(\mathrm{C}_{6} \mathrm{H}_{5} \mathrm{COCH}_{3}\right.$, MW 120) obtained on a tandem quadrupole instrument is shown in Figure 2a. The methane $\mathrm{CI}$ mass spectrum of this compound on the tandem quadrupole instrument is shown in Figure $2 \mathrm{~b}$. A small peak at $m / z 91$ is observed in this spectrum. Along with the $m / z 91$ ion there are a number of other ions in the spectrum that are also observed in the EI mass spectrum $(m / z 43,77,105$, and 120). The spectrum in Figure $2 b$ is the "best-case" spectrum that we could obtain, that is, the spectrum with the smallest $m / z$ 91. "The "real" methane CI mass spectrum (that resulting only from reactions of $\mathrm{CI}$ reagent ions with acetophenone) is shown in Figure 2c. Two ions constitute almost all of the signal, the protonated molecule at $\mathrm{m} / \mathrm{z} 121$ and the ion at $\mathrm{m} / \mathrm{z} 43$ resulting from loss of benzene from the protonated molecule. A small amount of fragmentation by loss of $\mathrm{CH}_{4}$ is also observed. This spectrum was obtained with a

\footnotetext{
*With the ion volume used for the spectrum shown in Figure 2 we obtained essentially the same relative intensities with a factor of 10 variation in sample pressure and also varying the reagent pressure $0.1-0.5$ torr (the maximum pressure at which the instrument could be operated). Using a different ion volume, the $\mathrm{m} / z 91$ peak in creased by about a factor of 6 . The other EI ions also increased significantly. Spectra with this ion volume were also reproducible over a time period of several months. These differences may be due to one or more parameters such as volume tightness or extraction field penetration.
}

quadrupole ion trap by trapping only the reagent ions $\left(\mathrm{CH}_{5}^{+}, \mathrm{C}_{2} \mathrm{H}_{5}^{+}, \mathrm{C}_{3} \mathrm{H}_{5}^{+}\right.$, and $\mathrm{H}_{3} \mathrm{O}^{+}$, the last being formed from residual water in the vacuum system) after the electron pulse and then allowing these ions to react with the analyte.

"Real" CI mass spectra can be obtained with the ion trap by trapping a range of ions [15] and letting all of the ions in this $m / z$ range react with the analyte. An alternative is to trap a single reagent ion, by use of RF and de potentials, and allow it to react with the analyte-the SRICI experiment. Individually selecting the reagent ions used to obtain the spectrum in Figure 2 c establishes that the fragment observed at $m / z 105$ comes only from reaction with $\mathrm{CH}_{5}^{+}$. This observation is consistent with the relative thermochemistry of the reagent ions, $\mathrm{CH}_{5}^{+}$providing the most exothermic proton transfer reaction. The spectrum in Figure $2 c$ can be "synthesized" from the spectra of the individual reagent ions if the relative intensities and relative reaction rates of the reagent ions are taken into account [16].

An alternative approach, for ion traps that cannot select a single reagent ion or a range of reagent ions because of lack of appropriate electronics ion trap detectors (ITDs) is to use a very low RF trapping level during the electron pulse. Under such operating conditions, higher mass ions are not efficiently trapped, and thus most EI-generated ions are not retained in the ion trap to react with the neutral reagent gas. The RF level is then raised after the ionization pulse to allow trapping of the higher mass products of the ion-molecule reactions of the reagent ions. In Figure $2 \mathrm{~d}$ the RF level during the electron pulse corresponded to a low mass cutoff of $3.6 \mathrm{u}$. Raising the low mass cutoff to $14.0 \mathrm{u}$ gives the results shown in Figure 2e. (The same spectrum is obtained as the RF level is increased up to the point at which the reagent ions are no longer trapped.) Notice that although there is evidence for a small amount of EI contribution in Figure $2 \mathrm{~d}$ from the increased relative intensity of $\mathrm{m} / \mathrm{z}$ 105 and appearance of $\mathrm{m} / \mathrm{z} 120$, Figure $2 \mathrm{e}$ has a significant intensity of ions not seen in Figure $2 c$ and d. Whereas in many instances the appearance of EI ions in the CI mass spectrum can actually be benefcial because they provide some structural information, the presence of an inn-molecule reaction product such as $m / z 91$ in Figure $2 b$ and e can lead to erroneous interpretation. Given the presence of ions corresponding to a phenyl ring $(m / z 77$ and $m / z 51)$, the most likely interpretation of $\mathrm{m} / z$ 91 would be that the precursor ion had a benzyl substructure. Assuming the foregoing, the most plausible interpretation of the spectra in Figure $2 b$ and $e$ would be that of propylbenzene.

Electron ionization ions contribute significantly to the total ion current in Figure $2 b$ and e. Although the analyte pressure and $\mathrm{CI}$ reaction time cannot be readily measured in the tandem quadrupole system, nor can the reaction time be easily varied, these parameters can be measured and controlled in the ion trap. 

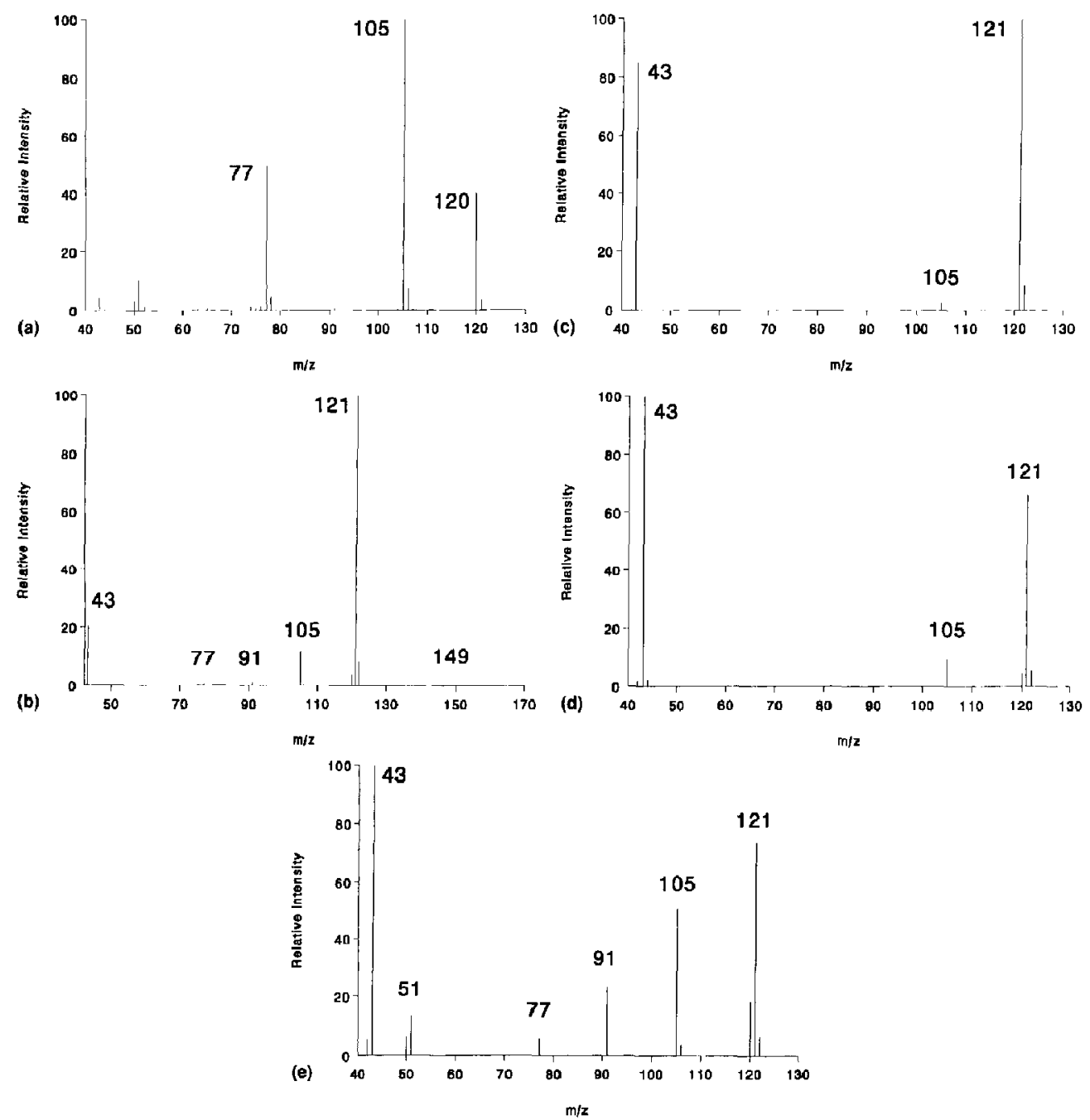

Figure 2. Mass spectrum of acetophenone acquired on the TSQ-700 using (a) EI and (b) methane CI (estimated source pressure 0.3 torr). The methane CI mass spectra acquired on the ITMS using (c) isolated methane reagent ions and a 50-ms reaction time, (d) methane $\mathrm{CI}$ with a low mass cutoff of $3.6 \mathrm{u}$, and (e) methane $\mathrm{CI}$ with a low mass cutoff of $14.0 \mathrm{u}$ are also shown. (Scans were started at $m / z 43$ to reject the CI reagent ions.)

This allows a reasonable analysis of what might be expected under various operating conditions. The number of EI-generated analyte ions can be approximated by eq 2 , and the number of $\mathrm{CI}$ analyte ions is approximated by eq 3 .

$$
\begin{aligned}
A^{+} \cdot & =e_{0}^{-}\left(1-e^{-\sigma l A}\right) \\
A H^{+} & =R_{0}^{+}\left(1-e^{-k t A}\right)
\end{aligned}
$$

In these equations, $e_{0}^{-}$is the number of ionizing electrons, $\sigma$ is the EI cross section under the ion trap operating conditions, $l$ is the path length of the electrons, $A$ is the analyte number density, $R_{0}^{+}$is the number of reagent ions, $k$ is the reaction rate constant, and $t$ is the reaction time. Using typical values for a CI experiment $-e_{0}^{-}=10^{10}, \sigma=10^{-15} \mathrm{~cm}^{2}, l=1$ $\mathrm{cm}, R_{0}^{\prime}=10^{7}, k=10^{10} \mathrm{~cm}^{3} /$ molecule-s, $t=20 \mathrm{~ms}$, and $A=10^{9}$ molecules $/ \mathrm{cm}^{3}$-it is expected that approximately similar intensities of $\mathrm{EI}$ and $\mathrm{Cl}$ ions should be formed. Actually, the rate constant for proton transfer may be somewhat greater (up to $3 \times 10^{-9}$ $\mathrm{cm}^{3} /$ molecule-s), which would lead to greater numbers of $\mathrm{Cl}$ ions being formed. Nevertheless, even in the best cases, the EI ions will still contribute noticeably to the $\mathrm{CI}$ mass spectrum. The two major variable parameters in the ion trap $\mathrm{Cl}$ experiment are the 
reagent gas pressure and reaction time. By increasing the reagent gas pressure, a shorter EI pulse is needed to generate $R_{0}^{+}$. A shorter electron pulse lowers the value of $e_{0}^{-}$in eq 2 and thus reduces the number of EI ions generated from the analyte. It should be noted, however, that significant increases in reagent gas pressure will adversely affect the performance of the ion trap by decreasing resolution and sensitivity [17] and will enhance the formation of products due to ion-molecule reactions with the reagent gas. Longer reaction times can have only marginal effects on $\mathrm{CI}$ sensitivity and may actually result in decreased sensitivity in certain experiments [16].

The foregoing discussion highlights how EIderived ions can appear in $\mathrm{CI}$ mass spectra obtained under some operating conditions in the ion trap. These EI ions can then react with the neutral reagent gas as shown in Figure 2e $(m / z$ 91). Given this, it should be no surprise that such processes can contribute significantly to EI spectra if alternate $\mathrm{EI}$ and $\mathrm{Cl}$ experiments are being performed with the ion trap. When methane is used as the $\mathrm{CI}$ reagent gas, the RF level during the electron pulse can readily be set such that no methane ions are trapped (e.g., low mass cutoff $>m / z 20$ ) and thus only EI-generated ions remain in the ion trap. However, if any EI ions react with methane, anomalous ions may appear in the mass spectrum. The ITMS mass spectra acquired for chlorobenzene, shown in Figure 3, are used to illustrate this point. The EI mass spectrum obtained when no methane was present in the ion trap is shown in Figure 3a, whereas the spectrum in Figure $3 b$ was acquired under identical conditions except methane was present in the ion trap at the pressure normally used for Cl. Although the signal intensity of $\mathrm{m} / \mathrm{z} 77$ is essentially equal to that of the base peak $\left(\mathrm{M}^{+}, m / z\right.$ 112 ) in the spectrum in Figure 3a, it is only about $50 \%$ of the base peak in Figure $3 \mathrm{~b}$, and the signal at $m / z$ 91, which is absent in Figure $3 a$, is also about $50 \%$ of the base peak in Figure $3 \mathrm{~b}$. The reaction of the ions at $m / z 77$ with $\mathrm{CH}_{4}$ occurs during the electron pulse and continues up to the time these ions are ejected from the ion trap. Thus in most cases, a significant portion of the "reaction time" will be during the analytical ramp used to obtain the mass spectrum. In Figure $3 \mathrm{~b}$, the analytical scan is started at an RF level corresponding to a low mass cutoff of $m / z 20$. The time from the start of the scan until ions at $\mathrm{m} / \mathrm{z} 77$ are ejected is $10.26 \mathrm{~ms}$. By starting the scan at a low mass cutoff of $m / z 50$, the reaction time during the scan is reduced to $4.86 \mathrm{~ms}$. This results in a reduction in the signal at $m / z 91$ by about $15 \%$ and an equivalent increase in the abundance of $m / z 77$. The change in abundance of these two ions allows the determination that $m / z$ is an ion-molecule reaction product of $m / z 77$. Thus the recognition of ion-molecule reactions when obtaining EI spectra in the presence of methane and the identification of reactant and product ions can be accomplished by simply taking two
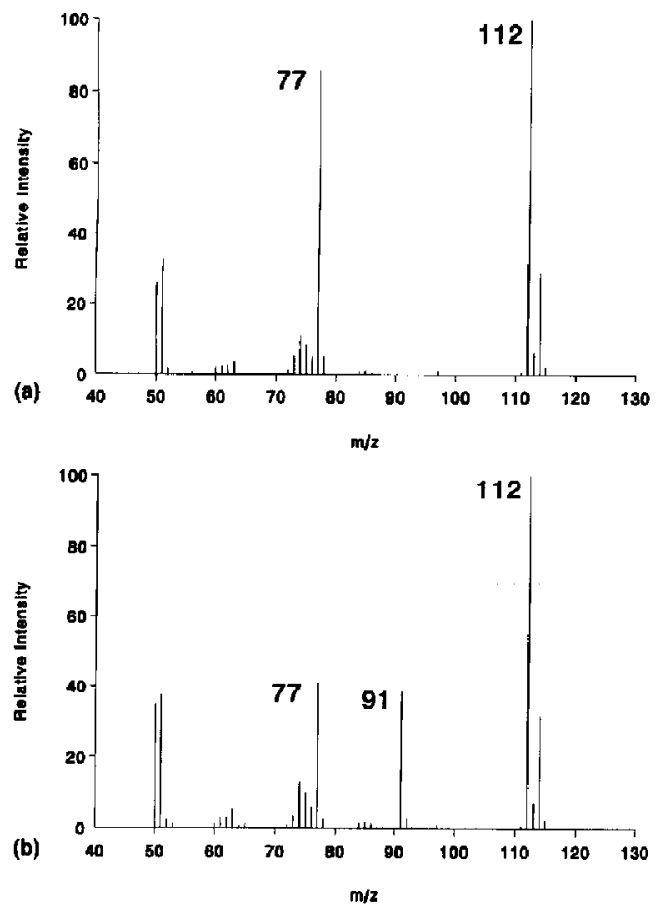

Figure 3. Electron impact mass spectrum of chlorobenzene acquired on the ITMS (a) without methane present and (b) with methane present.

spectra with different starting RF levels for the analytical scan.

Contributions to mass spectra from ion-molecule reactions such as this may be minimized by using a pulsed-valve inlet for the $\mathrm{CI}$ reagent gas rather than acquiring EI spectra in the presence of the reagent gas [18]. However, the full width at half maximum of a gas pulse can exceed $100 \mathrm{~ms}$, and thus it may be necessary to add some delay period between alternate scans to allow the pressure of the pulsed $\mathrm{CI}$ gas to be pumped down to pressures sufficiently low to minimize ion-molecule reactions.

\section{Reaction with Cl-generated Ions}

Whereas the quadrupole ion trap offers methods to eliminate reactions of El-generated ions with a $\mathrm{Cl}$ reagent gas, there is little that can be done to eliminate such reactions if a reactive ion is formed via $\mathrm{CI}$. The use of a pulsed valve for the reagent gas may reduce, but not eliminate, reactions of the reagent gas with reactive fragment ions due to the lifetime of the reagent gas within the trap, as mentioned above. The mass spectrum of chlorobenzene obtained when $\mathrm{CH}_{5}^{+}$ is selected as the reagent ion after the ionization pulse is shown in Figure 4. Contrary to the example with acetophenone, the signal at $m / z 91$ is present even after selecting the reagent ion subsequent to the elec- 


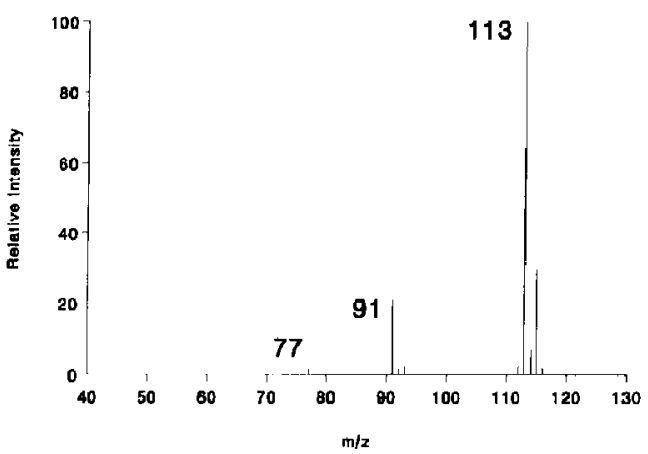

Figure 4. Mass spectrum of chlorobenzene acquired on the ITMS using $\mathrm{CH}_{5}^{+}$SRICI and a $30-\mathrm{ms}$ reaction time.

tron pulse. Previous high pressure methane $\mathrm{Cl}$ experiments with this compound did not show ions at $\mathrm{m} / \mathrm{z}$ 91 but did show ions at $m / z 93$ [13]. The latter ion was attributed to elimination of $\mathrm{HCl}$ from the collision complex of $\mathrm{CH}_{5}^{+}$and chlorobenzene. However, we believe that it is more likely to be the result of a collision-stabilized complex between $\mathrm{CH}_{4}$ and $\mathrm{C}_{6} \mathrm{H}_{5}^{+}$ (see below). In the $\mathrm{CI}$ mass spectrum obtained on the tandem quadrupole instrument shown in Figure 5, ions at $m / z 77, m / z 91$, and $m / z 93$ are all observed at a high pressure of methane in the source.

Although the formation of $\mathrm{C}_{6} \mathrm{H}_{5}^{+}$as an EI fragment cannot be precluded, the quadrupole ion trap data confurm that an ion at $m / z 77$ is formed as a CI fragment. Further substantiation that the ion at $\mathrm{m} / \mathrm{z}$ 77 is a CI fragment appears in Figure 6, which is a plot of the signals at $m / z 77$ and $m / z 91$ as a function of the reaction time before the analytical scan is started. At short reaction times, both ions are seen to increase in abundance. At about $15 \mathrm{~ms}$ the signal at $m / z 77$ reaches a maximum and then slowly declines in intensity. In contrast, the signal at $m / z 91$ continues to increase in intensity and is much greater in absolute intensity than the initial intensity of the ion $m / z 77$, thus requiring that ions at $m / z 77$ be formed

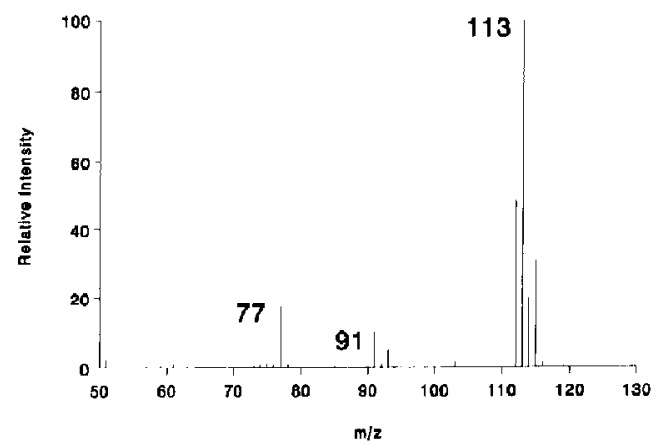

Figure 5. Methane CI mass spectrum of chlorobenzene acquired on the TSQ-700 with 0.5 torr estimated methane source pressures.

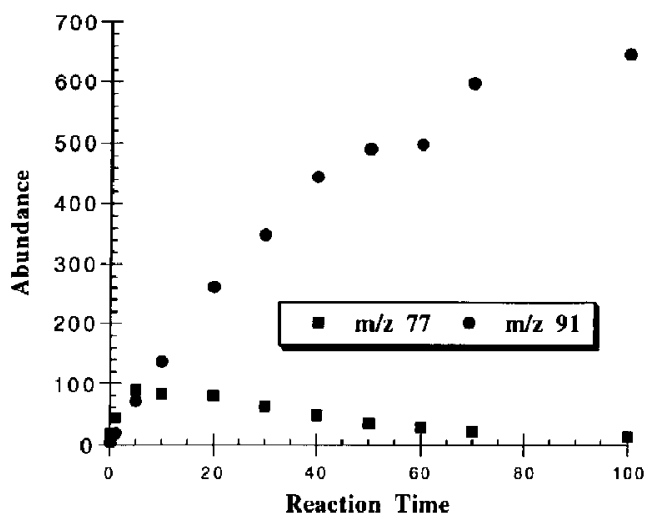

Figure 6. Plot of abundance of $m / z 77$ and $m / z 91$ versus reaction time in the ITMS.

during the reaction time. The intensity of $m / z 91$ starts to level off at reaction times greater than $\mathbf{5 0} \mathrm{ms}$ due to the significantly depleted reagent ion population at these reaction times, and thus little $\mathrm{m} / z 77$ is being formed. The overall process can be written as follows:

$$
\begin{gathered}
\mathrm{CH}_{5}^{+}+\mathrm{C}_{6} \mathrm{H}_{5} \mathrm{Cl} \rightarrow \mathrm{C}_{6} \mathrm{H}_{5} \mathrm{ClH}^{+}+\mathrm{CH}_{4} \\
\mathrm{C}_{6} \mathrm{H}_{5} \mathrm{ClH}^{+} \rightarrow \mathrm{HCl}+\mathrm{C}_{6} \mathrm{H}_{5}^{+} \\
\mathrm{C}_{6} \mathrm{H}_{5}^{+}+\mathrm{CH}_{4} \rightarrow \mathrm{C}_{7} \mathrm{H}_{9}^{+} \\
\mathrm{C}_{7} \mathrm{H}_{9}^{+} \rightarrow \mathrm{C}_{7} \mathrm{H}_{7}^{+}+\mathrm{H}_{2}
\end{gathered}
$$

The rates for reactions 5 and 7 will generally be too fast in the quadrupole ion trap experiment for thirdbody collisional stabilization, eq 8 .

$$
\left[\mathrm{C}_{7} \mathrm{H}_{9}^{+}\right]^{*}+\mathrm{CH}_{4} \rightarrow \mathrm{C}_{7} \mathrm{H}_{9}^{+}+\mathrm{CH}_{4}^{*}
$$

However, at higher pressures, as in the tandem quadrupole ion source, third-body collisions leading to stabilization may effectively compete with the dissociation channels. This would explain the observation of a signal at $m / z 93$ in the high pressure ion sources. Note that increasing the methane pressure increases the relative intensity of the $\mathrm{m} / z 93$ signal over that at $\mathrm{m} / \mathrm{z}$ 91. The increase in methane pressure has two effects. First, the number density of the reactant in eq 6 increases, resulting in increased formation of product (see eq 3). Second, the number density of $\mathrm{CH}_{4}$ in eq 8 is increased, leading to the stabilization of a greater percentage of $\mathrm{C}_{7} \mathrm{H}_{9}^{+}$.

Although reactions of $\mathrm{Cl}$-generated ions cannot be prevented in any type of instrument, the quadrupole ion trap again provides simple methods by which such reactions can be recognized compared to beam instruments. In the quadrupole ion trap, such reactions can be recognized by varying the $\mathrm{CI}$ reaction time. The reaction time can be readily varied by changing the starting point of the analytical scan as 
discussed earlier. A plot of the ratio of the abundances of $\mathrm{m} / \mathrm{z} 91$ to $\mathrm{m} / \mathrm{z} 77$ versus the reaction time prior to the start of the analytical scan (segment $B$ in Figure 1) for three different starting RF levels of the analytical scan is shown in Figure 7. Again, the change in ratio and direction of change at a given reaction time for different scan starting points indicates that ions at $\mathrm{m} / z$ 77 are reacting to produce ions at $\mathrm{m} / \mathrm{z}$ 91. Rather than changing the scan starting point, an alternative approach would be to change the $\mathrm{CI}$ reaction time. However, changing the starting point of the analytical scan is preferable because little or no new signal at $m / z 77$ is generated during the analytical scan. This results from the ejection of the reactant ion, $\mathrm{CH}_{5}^{+}$, which is generating the ion at $m / z 77$, at the start of the analytical scan or shortly thereafter. Therefore, at longer reaction times, when the initial reagent ion population has been significantly depleted and $\mathrm{C}_{6} \mathrm{H}_{5}^{+}$is formed at a lower rate, the ratio of $m / z 91$ to $m / z 77$ will be enhanced by allowing the ions at $m / z$ 77 present in the trap to react without producing any "replacement" $m / z 77$ ions. This trend is apparent in Figure 7. Shifting the data from scan start $=30$ up by $7.8 \mathrm{~ms}$ and the scan start $=50$ up by $3.6 \mathrm{~ms}$ (i.e., the difference in times before $m / z 77$ is ejected due to the scan range) gives similar ratios at shorter times. At longer times, however, the scan started at the lower RF value shows a greater ratio, although the difference is still less than when the starting point of the scan is changed.

\section{MS/MS-generated Ions}

Though the quadrupole ion trap provides several unique options for eliminating, or at least recognizing, ion-molecule reactions during $\mathrm{Cl}$, there are some potential disadvantages as well. Since the quadrupole

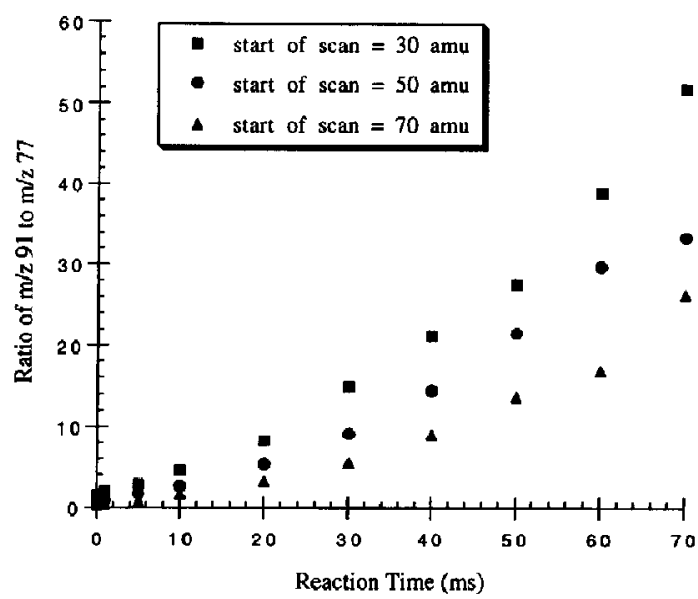

Figure 7. Plot of the ratio of $m / z 91$ to $m / z 77$ versus reaction time prior to the start of the scan ( $B$ in Figure 1) for analytical scans starting at 30,50 , and $70 \mathrm{u}$. ion trap performs all the stages of an experiment in the same analyzer, the $\mathrm{CI}$ reagent gas can affect other portions of the experiment in addition to the ionization step, such as reactions with product ions in MS/MS experiments. This rarely occurs in beam instruments because the region in which ionization takes place is physically separated, and usually differentially pumped, from the region in which the MS/MS reaction is effected, although there are cases in which such reactions have been observed [19].

We have previously discussed the reaction of $\mathrm{C}_{6} \mathrm{H}_{5}^{+}$ formed by CID of a molecule protonated via methane $\mathrm{CI}$ in a quadrupole ion trap [9]. Therefore, we present here a more complex case, one in which MS/MS product ions undergo competitive and consecutive reactions with the neutral reagent gas. In this example, the reactant species is a substituted phenyl cation, $\mathrm{C}_{6} \mathrm{H}_{4} \mathrm{Cl}^{+}(m / z 111)$ derived from $p$-dichlorobenzene. The CID product ion spectrum $(30 \mathrm{eV})$ of the $(\mathrm{M}+$ $\mathrm{H})^{+}$ion of $p$-dichlorobenzene, $m / z$ 147, obtained on the TSQ has $m / z 111$ (loss of $\mathrm{HCl}$ ) as the only product ion formed. The ion trap CID spectrum of the same ion is shown in Figure 8a. Note the presence of the ion-molecule reaction product ion, $m / z$ 91, in the spectrum with about $50 \%$ of the abundance of the major CID product, $m / z$ 111. This ion-molecule reaction proceeds during the application of the auxiliary $\mathrm{RF}$ "tickle" voltage, which induces CID in the ion trap, and during the analytical ramp as shown earlier in this discussion. The overall process can be written as

$$
\begin{gathered}
\mathrm{CH}_{5}^{+}+\mathrm{C}_{6} \mathrm{H}_{4} \mathrm{Cl}_{2} \rightarrow \mathrm{C}_{6} \mathrm{H}_{4} \mathrm{Cl}_{2} \mathrm{H}^{+}+\mathrm{CH}_{4} \\
\mathrm{C}_{6} \mathrm{H}_{4} \mathrm{Cl}_{2} \mathrm{H}^{+} \rightarrow \mathrm{HCl}+\mathrm{C}_{6} \mathrm{H}_{4} \mathrm{Cl}^{+}(\mathrm{CID}) \\
\mathrm{C}_{6} \mathrm{H}_{4} \mathrm{Cl}^{+}+\mathrm{CH}_{4} \rightarrow \mathrm{HCl}+\mathrm{C}_{7} \mathrm{H}_{7}^{+}
\end{gathered}
$$

The spectrum resulting when an additional $20 \mathrm{~ms}$ of reaction time subsequent to the tickle pulse was incorporated into the scan function is shown in Figure $8 \mathrm{~b}$. Note that the signal at $m / z 91$ is the base peak, and the intensity of the signal at $m / z 111$ has substantially decreased. There is also evidence of other ion-molecule reactions occurring due to the presence of ions at $m / z 79, m / z 105$, and $m / z 125$. Another 20 $\mathrm{ms}$ of reaction time was added to the ion trap scan function (a delay of $40 \mathrm{~ms}$ after the RF tickle pulse) to determine if any further ion-molecule reaction products would be observed. The spectrum obtained using this scan function is provided in Figure $8 \mathrm{c}$. An additional ion-molecule reaction product was observed at $\mathrm{m} / \mathrm{z} 107$. A plot of the relative intensity of the ions found in the spectra of Figure 8 versus reaction time after the RF tickle pulse is provided in Figure 9. Note that the $m / z 91$ signal eventually reaches a maximum and then slowly decreases as the other ion-molecule reactions occur. Thus the $m / z 91$ ion is an ion-molecule reaction product of the CID product ion, $\mathrm{m} / z$ 111. The $m / z 105, m / z 107$, and $m / z 79$ ions are 

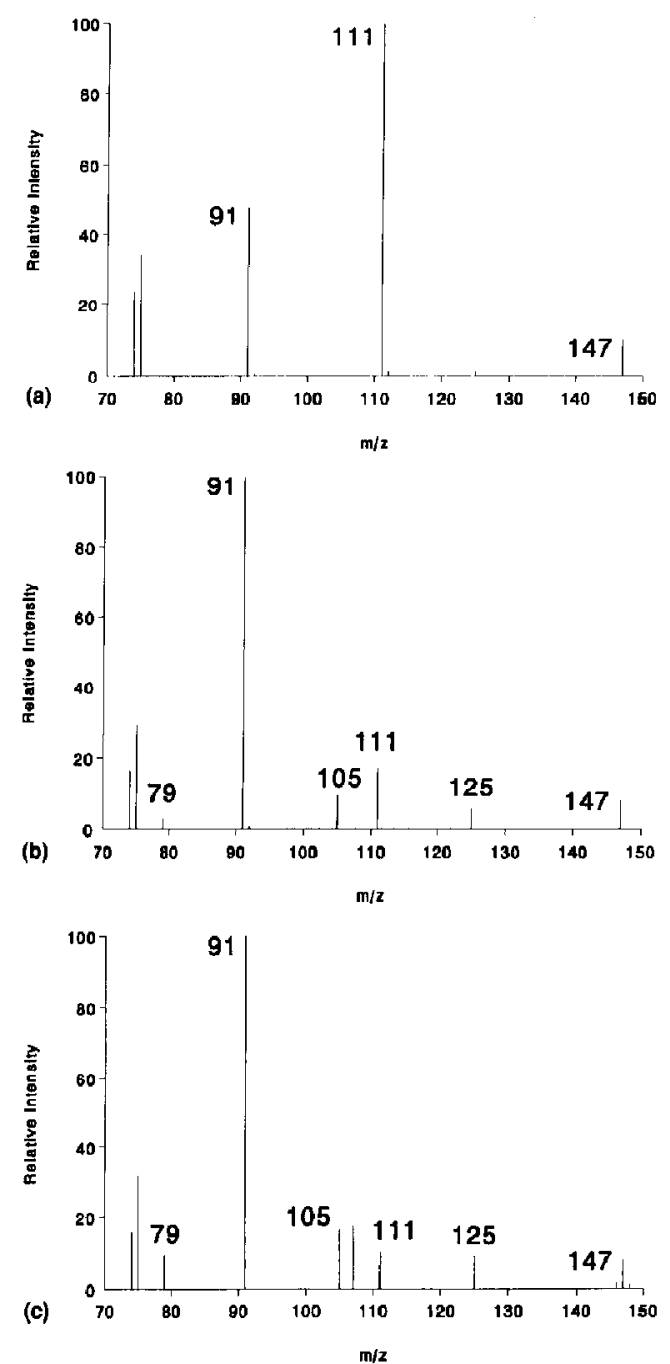

Figure 8. MS/MS spectrum of the $(\mathrm{M}+\mathrm{H})^{+}$ion of $p$-dichlorobenzene acquired on the ITMS with (a) no additional reaction time, (b) $20 \mathrm{~ms}$ additional reaction time, and (c) $40 \mathrm{~ms}$ additional reaction time incorporated into the scan function after the tickle pulse.

ion-molecule reaction products of $m / z 91$ ions, and the ion at $m / z 125$ is formed by reaction of $\mathrm{C}_{6} \mathrm{H}_{4} \mathrm{Cl}^{+}$. These relationships were confirmed by performing a "double-resonance" experiment [20]. The tickle voltage was used to eject ions of a selected $\mathrm{m} / \mathrm{z}$ from the ion trap during the reaction period, in which case the next-generation ion-molecule reaction products could not be produced when the ejected ions are the reactant ions.

The ions at $m / z 125$ are formed by a reaction analogous to eq 11 except that $\mathrm{H}_{2}$ is the neutral lost instead of $\mathrm{HCl}$. The ions at $m / z 107$ and 105 are generated by reactions analogous to eq 1 and eqs 6 and 7 , respectively. The ion at $m / z 79$ results from

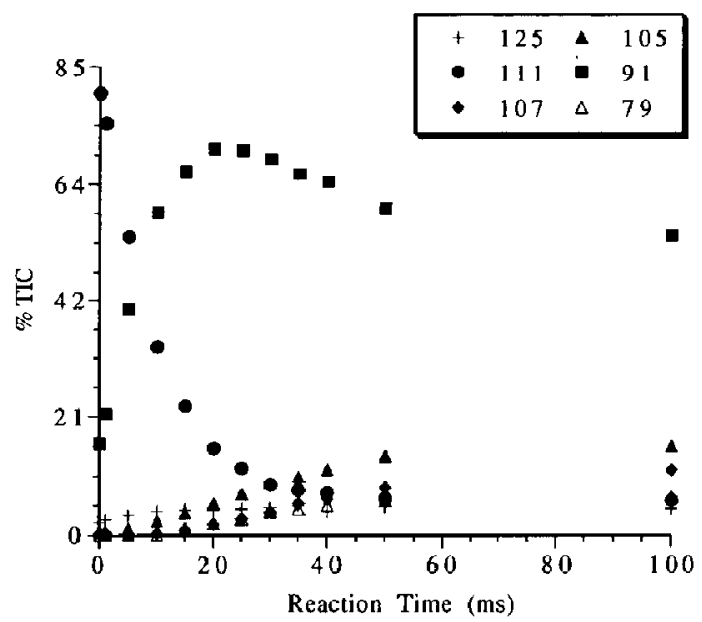

Figure 9. Plot of the relative abundance of CID and ion-molecule reaction products observed in the MS/MS spectrum of the $(\mathrm{M}+\mathrm{H})^{+}$ion of $p$-dichlorobenzene versus reaction time in the ITMS (\% TIC $=$ percent total ion current).

loss of $\mathrm{C}_{2} \mathrm{H}_{4}$ from $\mathrm{m} / z 107$ and is competitive with eq 7. The fact that the $m / z 91$ abundance eventually levels off at longer reaction times is indicative of the presence of two isomeric ions with a nominal massto-charge ratio of 91 . One of these ions is reactive with methane; the other is not. The chemistry of these reactions will be presented elsewhere [12].

\section{Conclusions}

Reactions of $\mathrm{CI}$ reagent gas with ions generated by various means can occur in $\mathrm{CI}$ in conventional beam instrument sources and low pressure ion trap experiments. Whereas little can be done to alleviate or even recognize these reactions in the higher pressure ion sources used in beam instruments, the ion trap mass spectrometer affords simple methods for establishing the presence of ion-molecule reaction products and, in certain instances, minimizing or precluding such reactions in EI and CI spectra. The most general method for recognizing such reactions in the ion trap is to vary the starting point of the analytical ramp. Any changes in the relative intensity of the ions in the spectra acquired at different starting points for the analytical ramp are likely due to ion-molecule reactions. Selected reagent ion chemical ionization can be used to eliminate ions produced during the electron pulse that are not reagent ions, thus avoiding possible reactions of these ions with the $\mathrm{Cl}$ reagent gas. Ionmolecule reactions with reactive ions formed by CID of ions generated by $\mathrm{Cl}$ in the ion trap are difficult to avoid because the two processes take place within the same volume. It is possible, however, to identify which ions are ion-molecule products by placing additional reaction time after the CID step in the ion trap 
scan function or by varying the starting point of the analytical ramp.

\section{Acknowledgments}

Research sponsored by the U.S. Department of Energy, Office of Basic Energy Sciences, under Contract DE-AC05-84OR21400 with Martin Marietta Energy Systems, Inc. KJH acknowledges an appointment to the U.S. Department of Energy, Laboratory Cooperative Postgraduate Research Training Program administered by Oak Ridge Associated Universities.

\section{References}

1. Harrison, A. G. Chemical Ionization Mass Spectrometry; CRC Press: Boca Raton, FL, 1983.

2. (a) Van Berkel, G. J.; Glish, G. L.; McLuckey, S. A.; Tuinman, A. A. J. Am. Chem. Soc. 1989, 111, 6027. (b) McEwen, C. N.; Rudat, M. A. J. Am. Chem. Sac. 1979, 101, 6470. (c) Butdzikiewicz, H. Org. Mass Spectrom. 1988, 23, 561. (d) Brophy, J. J.; Diakiw, V.; Goldsack, R. J.; Nelson, D.; Shannon, J. S. Org. Mass Spectrom. 1979, 14, 201. (e) Sears, L. J.; Campbell, J. A.; Grimsrud, E. P. Biomed. Environ. Mass Spectrom. 1987, 14, 401.

3. Bonner. R, F.; Lawson, G.; Todd, J. F. J. I. Chem. Soc. Chem. Commun, 1972, 1179. (b) Brodbelt, J. S.; Louris, J. N.; Cooks, R. G. Anal. Chem. 1987, 59, 1278. (c) Clow, R. P.; Futrell, J. H. I. Am. Chem. Soc. 1972, 94, 3748.

4. Bartmess, J. E.; Georgiadis, R. M. Vacuum 1983, 33, 149.

5. Field, F. H. In: Ion-Molecule Reactions; Franklin, J. L., Ed.; Plenum Press: New York, 1972, vol 1.
6. Glish, G. L.; Van Berkel, G. J,; McLuckey, S. A. Adv. Mass Spectrom. 1989, 11, 596.

7. Louris, J. N.; Cooks, R. G.; Syka, J. E. P.; Kelley, P. L.; Stafford Jr., G. C.; Todd, J. F. J. Anal. Chem. 1987, 59, 1677.

8. Speranza, M.; Sefcik, M. D.; Henis, J. M. S.; Gaspar, P. P. J. Ant. Chem. Soc. 1977, 99, 5583.

9. Lifshitz, C.; Gibson, D.; Levsen, K. Int. I. Mass Spectrom. Ion Proc. 1980, 35, 365.

10. McLuckey, S. A.; Glish, G. L., Van Berkel, G. I. Int. J. Mass Spectrom. Ion Proc. 1991, 106, 213.

11. Ausloos, P.; Lias, S. G.; Buckley, T. J.; Rogers, E. E. Int. J. Mass Spectrom. Ion Prik. 1989, 92, 65.

12. Hart, K. J.; McLuckey, S. A.; Glish, G. L. in preparation.

13. Leung, H.-W.; Harrison, A .G. Can. J. Chem. 1976, 54 , 3439.

14. Bone, L. I.; Futrell, J. H. J. Chem. Phys. 1967, 46, 4084.

15. McLuckey, S. A.; Goeringer, D. E.; Glish, G. L. J. Am. Soc. Mass Spectrom. 1991, 2, 11.

16. Eckenrode, B. A.; McLuckey, S. A.; Glish, G. L. Int. I. Mass Spectrom. Ion Proc. 1991, 106, 137.

17. Stafford Jr., G. C.; Kelley, P. E.; Syka, J. E. P.; Reynolds, W. E.; Todd, J. F. J. Int. J. Mass Spectrom. Ion Proc. 1984, 60,85 .

18. Emary, W. B.; Kaiser, R. E.; Kenttämaa, H. I.; Cooks, R. G. J. Am. Soc. Mass Spectrom. 1990, $1,308$.

19. Dolnikowski, G. G.; Heath, T. G.; Watson, J. T.; Scrivens, J. H.; Rolando, C. H. I. Am. Soc. Mass Spectrom. 1990, 1, 481.

20. Ander, L. R.; Beauchamp, J. L.; Dunbar, R. C.; Baldeschwieler, J. D. J. Chem. Phys. 1966, 45, 1062. 